\title{
Low-Frequency rTMS and Intensive Occupational Therapy Improve Upper Limb Motor Function and Cortical Reorganization Assessed by Functional Near-Infrared Spectroscopy in a Subacute Stroke Patient
}

\author{
Naoki Urushidani ${ }^{a}$ Shoji Kinoshita ${ }^{b, c}$ Takatsugu Okamoto ${ }^{a, b}$ \\ Hiroaki Tamashiro ${ }^{a}$ Masahiro Abo ${ }^{b}$
}

${ }^{a}$ Nishi-Hiroshima Rehabilitation Hospital, Hiroshima, Japan; ${ }^{b}$ Department of Rehabilitation Medicine, The Jikei University School of Medicine, Tokyo, Japan; 'Department of Rehabilitation Medicine, Aomori Shintoshi Hospital, Aomori, Japan

\section{Keywords}

Functional near-infrared spectroscopy $\cdot$ Kaifukuki rehabilitation wards $\cdot$ Repetitive transcranial magnetic stimulation - Occupational therapy · Rehabilitation

\section{Abstract}

There is still no agreement on the most suitable time and modality for application of repetitive transcranial magnetic stimulation (rTMS) to improve motor recovery in subacute stroke patients. The underlying mechanism of motor recovery following low-frequency rTMS is considered to be modulation of the interhemispheric asymmetry. On the other hand, the cortical balance of brain activity during the acute to chronic phase of stroke is reported to be unstable. 


\section{Case Reports in Neurology}

Therefore, we conducted this study to clarify the time course of the interhemispheric asymmetry and the effect of application of low-frequency rTMS combined with occupational therapy on motor recovery and cortical imbalance of brain activity in a subacute stroke patient. The interhemispheric asymmetry in this patient with new-onset subcortical cerebral infarction and upper limb hemiparesis was evaluated longitudinally using functional near-infrared spectroscopy with finger tasks. A nonlesional hemisphere-dominant activation pattern was observed on day 28 after onset. On day 56 after onset, a bilaterally eminent activation pattern was observed. Low-frequency rTMS was applied on day 109 after stroke onset when the cortical activity shifted to the nonlesional hemisphere. The treatment resulted in improvement in motor function of the affected upper limb and a shift in brain activation to the lesional hemisphere. Our report is the first to describe the therapeutic benefits of low-frequency rTMS as assessed by longitudinal neuroimaging for functional recovery and interhemispheric asymmetry in a subacute stroke patient.

(C) 2018 The Author(s)

Published by S. Karger AG, Basel

\section{Introduction}

Repetitive transcranial magnetic stimulation (rTMS) was introduced recently as a therapeutic modality for poststroke upper limb hemiparesis. The combined application of low-frequency rTMS (LF-rTMS) over the nonlesional cerebral hemisphere and intensive occupational therapy (OT) was demonstrated by several groups to be effective treatment for motor dysfunction affecting the upper limbs in chronic poststroke patients $[1,2]$. The underlying mechanism of motor recovery following LF-rTMS/OT is speculated to be related to the modulation of this interhemispheric imbalance (IHI) [3]. On the other hand, the interhemispheric balance of brain activity during the acute to chronic phase of stroke is reported to be unstable $[4,5]$. Based on these facts, it would be reasonable to assess the progression of IHI before the application of the LF-rTMS/OT and its effect on motor recovery in subacute stroke.

Functional near-infrared spectroscopy (fNIRS) has been used increasingly in studies of neurorehabilitation, including stroke [6]. The NIRS system measures noninvasively the hemodynamic changes in tissues, such as the cortical microvessels, by detecting the characteristic absorption spectra of hemoglobin through near-infrared light probes and, thus, provides assessment of regional hemodynamic responses associated with cortical brain activation. The use of fNIRS for the assessment of cortical neural activity yields results similar to those obtained by functional magnetic resonance imaging (fMRI) [6]. However, to date, there is no consensus on the changes in brain activation patterns relative to recovery of upper limb function.

The present study was designed to clarify the time course of interhemispheric asymmetry in a patient with subacute stroke and the effects of application of LF-rTMS/OT on the cortical imbalance of brain activity, as assessed by fNIRS. For this purpose, we conducted the present study using longitudinal fNIRS measurements to evaluate the IHI in a patient with subacute cerebral infarction and upper limb hemiparesis. 


\section{Case Reports in Neurology}

Case Rep Neurol 2018;10:223-231

DOI: $10.1159 / 000492381$

(C) 2018 The Author(s). Published by S. Karger AG, Base www.karger.com/crn

Urushidani et al.: LF-rTMS/OT with Longitudinal fNIRS

\section{Case Presentation}

The present study was conducted in compliance with the Declaration of Helsinki. The study protocol and intervention were granted approval by the Ethics Committee of Nishi-Hiroshima Rehabilitation Hospital, and informed consent was obtained from the patient before entry into the study. In addition, based on the previous evidence-based guidelines for rTMS [1], the subacute phase of stroke was defined as the period of time after 1-3 weeks after stroke onset until 6 months after stroke onset in this study.

A 71-year-old woman suffered sudden right hemiparesis and was transferred to an acute hospital about $7 \mathrm{~h}$ after the onset. Based on MRI with a high-intensity area in the left putamen, the patient was diagnosed with cerebral infarction and received conservative antiplatelet therapy. On day 17 after onset, the patient was transferred to the convalescent rehabilitation ward at Nishi-Hiroshima Rehabilitation Hospital for multidisciplinary rehabilitation. At the time of admission to this hospital, she was completely alert with noticeable but mild rightsided hemiparesis. She needed assistance for standing, walking, and toileting. She could flex and extend her finger in the affected side. However, she was not able to pinch or grip with her affected fingers. The Fugl-Meyer Assessment (FMA) score for the upper limbs was 30. Furthermore, the Action Research Arm Test (ARAT) was 6 (Table 1), and the Functional Independence Measure (FIM) was 109. The FMA is a performance-based quantitative measure for the assessment of various impairments in poststroke patients, with assessment of upper limb motor function comprising 33 items of measurement. The maximum possible motor performance score for upper limbs is 66 points. The ARAT consists of 19 functional movement tasks divided into 4 domains (grasp, grip, pinch, and gross movement). The maximum summed score is 57 . The FIM is a standardized and widely used tool for assessment of activity of daily living. The maximum score of FIM is 126.

After the admission to the convalescent rehabilitation ward, the patient received physical therapy and OT, which focused mainly on walking ability and the activity of daily living. In addition, serial assessments of motor function of the affected upper limb with FMA and ARAT and brain activity with fNIRS were conducted.

A 49-channel fNIRS system (FOIRE-3000, Shimadzu, Kyoto, Japan) was used to record cortical activity. The task used during fNIRS involved repetitive movements of flexion and extension of the paretic fingers, using a block paradigm design (3 cycles; resting [15 s] - hand movements [15 s] - resting [15 s]). Based on the modified Beer-Lambert law, we acquired changes in oxyhemoglobin concentration $(\Delta \mathrm{Oxy}-\mathrm{Hb})$ at baseline. $\Delta \mathrm{Oxy}-\mathrm{Hb}$ levels were selected in this study since they were found to be reliable and sensitive to changes in cerebral blood flow [6]. The locations of the optodes and the anatomical landmarks were marked using a 3D digitizer (FASTRAK, Polhemus, VT, USA). The Statistical Parametric Mapping software package (NIRS-SPM, Welcome Trust Centre for Neuroimaging, London, UK) was used to analyze fNIRS data. The NIRS-SPM was used for spatial registration of the acquired channels on the Montreal Neurological Institute (MNI) brain. Gaussian smoothing with full width at half maximum of $2 \mathrm{~s}$ was applied to estimate and remove temporal autocorrelation. The wavelet minimum description length-based detrending algorithm was used to correct any signal distortion. General linear model analysis with a hemodynamic response curve to model the hypothesized oxygenated hemoglobin ( $\mathrm{HbO}$ ) response during the tasks was performed. Based on the general linear model analysis, topography was plotted based on the location of the 


\section{Case Reports in Neurology}

channels. When SPM t-statistic maps were computed, $\mathrm{HbO}$ was considered significant at an uncorrected threshold of $p<0.01$. The primary sensory-motor cortex (Brodmann area; BA4) and premotor cortex and supplementary motor area (BA6) were selected as regions of interest. This selection was based on previous studies indicating that BA6 plays an important role in functional recovery after motor impairment $[7,8]$. Two appropriate channels corresponding to each region of interest were defined using MNI coordinates of the channels. The $\Delta 0 x y-$ $\mathrm{Hb}$ of 4 channels during the task phases was averaged, resulting in a $\Delta 0 \mathrm{xy}-\mathrm{Hb}$ signal for the left and right BA4 + BA6 areas. To assess the imbalance of cortical activity, the laterality index (LI) was analyzed for the averaged $\Delta 0 \mathrm{xy}-\mathrm{Hb}$ in the BA4 + BA6 areas. LI was defined as [( $\Delta \mathrm{Oxy}-$ $\mathrm{Hb}$ in affected hemisphere $-\Delta \mathrm{Oxy}-\mathrm{Hb}$ in unaffected hemisphere $) /(\Delta \mathrm{Oxy}-\mathrm{Hb}$ in affected hemisphere $+\Delta \mathrm{Oxy}-\mathrm{Hb}$ in unaffected hemisphere)]. LI of +1 and -1 represented activation in the lesional and nonlesional hemispheres, respectively.

The scores of both the FMA and ARAT at 2 months after onset showed improvement relative to those recorded at admission (Table 1). In addition, the LI for BA4 + BA6 was also increased (Table 1; Fig. 1b). During the same time period, the patient demonstrated independence in activity of daily living by using a wheelchair. She was able to grip and pinch with affected finger. However, she did not use her affected upper limb in the activity of daily living. In comparison, during the period from 2 to 4 months after onset, the motor function of the affected limb showed no improvement and the LI decreased (Table 1; Fig. 1c). During that time period, the patient achieved walking independence. Based on these changes, we decided to administer the 15-day LF-rTMS/OT course at day 109 after onset. During the 15-day therapy, the subject received 21 treatment sessions of daily 20-min LF-rTMS and 120-min intensive OT ( 2 sessions per day).

The low-frequency rTMS was applied using a 70-mm F-8 coil and MagPro R30 stimulator (MagVenture Company, Farum, Denmark). In each session, rTMS of 1,200 pulses at $1 \mathrm{~Hz}$ was applied on the skull of the nonlesional hemisphere at a site that elicited the largest motorevoked potentials in the first dorsal interosseous muscle of the unaffected upper limb using surface electromyography. The stimulation intensity was set at $90 \%$ of the motor threshold of the first dorsal interosseous muscle.

A 60-min one-on-one training was introduced on an individual basis in a face-to-face fashion by an occupational therapist. This training mainly involved the application of shaping techniques and repetitive task practice techniques. The shaping techniques concentrated on the use of the affected upper limb in functional tasks for activities of daily living. We provided repeated combined motion training using various objects, similar to those commonly used in the activity of daily living. A 60-min self-exercise was encouraged in another quiet room without any supervision after the intensive OT. The occupational therapist provided the self-exercise program that met the needs of the patient after discussing the program content with the patient.

The scheduled 15-day protocol of LF-rTMS/OT was completed by the patient without any adverse events. As shown in Table 1, the scores on FMA and ARAT improved after the intervention. The LI for BA4 + BA6 areas also increased (Fig. 1d). In addition, the patient was able to cut vegetables with a knife and manipulate chopsticks held by the hand on the affected upper limb. 


\section{Case Reports in Neurology}

Case Rep Neurol 2018;10:223-231

DOI: $10.1159 / 000492381$

(C) 2018 The Author(s). Published by S. Karger AG, Base www.karger.com/crn

Urushidani et al.: LF-rTMS/OT with Longitudinal fNIRS

\section{Discussion}

In the present study, the progression of interhemispheric asymmetry was followed in a subacute stroke patient who received interdisciplinary rehabilitation therapy, using fNIRS. The combination treatment of LF-rTMS/OT was applied and completed safely in a stroke patient at 4 months after onset, based on the fact that cortical activation was shifted to the nonlesional hemisphere, as confirmed by fNIRS. The 15-day LF-rTMS/OT treatment increased motor function of the affected limb and facilitated a shift in brain activation to the lesional hemisphere.

The positive effect of rTMS on poststroke recovery of functional motor activity has been well established [1,2], but the debate on the most suitable time and modality for application of LF-rTMS for subacute stroke patients continues to date. Another previous study showed that application of high-frequency rTMS (HF-rTMS) of $\geq 5 \mathrm{~Hz}$ activated local neural excitability, whereas LF-rTMS of $\leq 1 \mathrm{~Hz}$ suppressed local neural excitability [9]. The efficacy of LF-rTMS applied to the nonlesional motor cortex for chronic stroke was highlighted by the current evidence-based guideline [1]. We reported previously that the protocol of LF-rTMS/OT significantly improved the motor function of the affected upper limb in chronic stroke patients [2]. On the other hand, for acute stroke, Sasaki et al. [10] reported that HF-rTMS of the lesional hemisphere was more effective than LF-rTMS of the nonlesional cortex for motor recovery of the upper limbs. For subacute stroke, Hosomi et al. [11] examined the clinical effects of HFrTMS on upper limb motor function of subacute stroke patients in a randomized controlled trial and reported significant improvement only in the Brunnstrom stages hand score compared to sham stimulation. However, no improvements were noted in Brunnstrom stages upper limb scores and FMA after rTMS compared to sham. However, to our knowledge, there was no clinical study yet that clarified the effect of LF-rTMS on functional motor recovery in subacute stroke.

With regard to neural reorganization after stroke onset, it is conceivable that the application of rTMS can be decided on based on the neuroimaging findings in subacute stroke. The abnormally high interhemispheric inhibition in patients with chronic stroke is reported to be associated with poor recovery of hand motor performance [12]. In this regard, the underlying mechanism of the effect of LF-rTMS is mainly functional reorganization in the lesional hemisphere, since suppressive LF-rTMS over the nonlesional hemisphere reduces interhemispheric inhibition toward the lesional hemisphere and indirectly activates the lesional hemisphere [3]. Actually, Yamada et al. [8] performed fMRI before and after the application of LFrTMS/OT in a group of chronic stroke patients and reported that the intervention induced a shift in bilateral brain activation to the affected hemisphere in the primary sensorimotor cortex. On the other hand, imbalance in cortical brain activity is known to be unstable in subacute stroke. Previous studies investigated the time course of cortical activation by using fMRI after stroke onset and reported a change in interhemispheric asymmetry of cortical activation from the acute to chronic phase $[4,5]$. Considered together, it would be necessary to assess the progression of IHI before the application of LF-rTMS in the functional recovery of subacute stroke patients. To our knowledge, there is no clinical study that examined the time course of IHI and clinical effect of LF-rTMS on the functional outcome and IHI using fNIRS measurement. Therefore, our report describes the first clinical study that examined the safety and feasibility 
of LF-rTMS with longitudinal neuroimaging in the functional recovery of a subacute stroke patient.

In the present study, during the period between the 56th and 109th day, we found a shift of the activation to the nonlesional hemisphere and no improvement in motor function of the affected limb. In subacute stroke patients with subcortical lesions, Wei et al. [13] performed serial fMRI measurements and found an increase in cortical activation in the contralesional nonprimary motor cortex after the rehabilitative training. Our results are consistent with the findings of the aforementioned study where a shift of activation was found after rehabilitation therapy. In addition, a possible reason why there was no functional improvement in the affected upper limb during that phase is that the progression of IHI might offset the effect of rehabilitation therapy. However, our study does not provide a clear reason for the progression of IHI in subacute stroke. It is necessary to clarify the mechanism of the shift in brain activation to the nonlesional hemisphere and its influence on motor recovery in a future study.

\section{Limitations}

First, the clinical effectiveness was not studied in this case report. Further studies are needed to confirm the effectiveness of LF-rTMS/OT in patients with subacute stroke based on the findings of functional neuroimaging that can provide accurate assessment of IHI. Second, we could not examine the appropriate rTMS modality for patients without excessive IHI. Based on the presumed mechanism of rTMS, the application of HF-rTMS to the injured cerebral hemisphere might be more effective than application of LF-rTMS to the noninjured hemisphere for motor recovery in patients without severe IHI in the cerebral cortex. Further prospective studies are needed to confirm the usefulness of this neuroimaging-based rTMS therapy for upper limb hemiparesis.

\section{Conclusions}

The present case report described the progression of interhemispheric asymmetry in a patient with subacute stroke using longitudinal fNIRS measurements. In addition, the timing of application of LF-rTMS/OT was set to coincide with a shift in cortical activity to the nonlesional hemisphere. The applied treatment improved upper limb motor function and facilitated a shift in brain activation to the lesional hemisphere. Our report describes the first clinical study that examined the feasibility and safety of LF-rTMS using longitudinal neuroimaging for assessment of functional recovery and progression of interhemispheric asymmetry in a patient with subacute stroke.

\section{Acknowledgments}

This work was supported by Japan Society for the Promotion of Science KAKENHI, grant No. 16K16443. On behalf of the NEURO investigators of the Nishi-Hiroshima Rehabilitation Hospital, the authors gratefully acknowledge the support and participation of the patient in 
the present study. The NEURO investigators of the Nishi-Hiroshima Rehabilitation Hospital are Takatsugu Okamoto, MD, PhD; Eiji Inoue, OTR, MS; Sachiko Shiraoka, OTR; Naoki Urushidani, OTR; Hiroaki Tamashiro, OTR; Takashi Kurushima, OTR; Takashi Matsuda, OTR; Makoto Murakami, OTR; Chiho Oka, OTR; Nozomi Takagi, OTR; Shinya Osako, OTR; Yumiko Okuda, OTR; Takuya Ojimo, OTR; Haruna Okada, OTR; Haruka Ode, OTR; and Chihiro Murakami, OTR.

\section{Statement of Ethics}

The present study was conducted in compliance with the Declaration of Helsinki. The study protocol and intervention were granted approval by the Ethics Committee of Nishi-Hiroshima Rehabilitation Hospital, and informed consent was obtained from the patient before entry into the study.

\section{Disclosure Statement}

The authors report no conflicts of interest. The authors alone are responsible for the contents and writing of this paper.

\section{References}

1 Lefaucheur JP, André-Obadia N, Antal A, Ayache SS, Baeken C, Benninger DH et al. Evidence-based guidelines on the therapeutic use of repetitive transcranial magnetic stimulation (rTMS). Clin Neurophysiol. 2014 Nov;125(11):2150-206.

2 Abo M, Kakuda W, Momosaki R, Harashima H, Kojima M, Watanabe S et al. Randomized, multicenter, comparative study of NEURO versus CIMT in poststroke patients with upper limb hemiparesis: the NEUROVERIFY Study. Int J Stroke. 2014 Jul;9(5):607-12.

3 Corti M, Patten C, Triggs W. Repetitive transcranial magnetic stimulation of motor cortex after stroke: a focused review. Am J Phys Med Rehabil. 2012 Mar;91(3):254-70.

4 Marshall RS, Perera GM, Lazar RM, Krakauer JW, Constantine RC, DeLaPaz RL. Evolution of cortical activation during recovery from corticospinal tract infarction. Stroke. 2000 Mar;31(3):656-61.

5 Askim T, Indredavik B, Vangberg T, Håberg A. Motor network changes associated with successful motor skill relearning after acute ischemic stroke: a longitudinal functional magnetic resonance imaging study. Neurorehabil Neural Repair. 2009 Mar-Apr;23(3):295-304.

6 Miyai I, Tanabe HC, Sase I, Eda H, Oda I, Konishi I et al. Cortical mapping of gait in humans: a near-infrared spectroscopic topography study. Neuroimage. 2001 Nov;14(5):1186-92.

7 Calautti C, Baron JC. Functional neuroimaging studies of motor recovery after stroke in adults: a review. Stroke. 2003 Jun;34(6):1553-66.

8 Yamada N, Kakuda W, Senoo A, Kondo T, Mitani S, Shimizu M et al. Functional cortical reorganization after low-frequency repetitive transcranial magnetic stimulation plus intensive occupational therapy for upper limb hemiparesis: evaluation by functional magnetic resonance imaging in poststroke patients. Int J Stroke. 2013 Aug;8(6):422-9.

9 Fitzgerald PB, Fountain S, Daskalakis ZJ. A comprehensive review of the effects of rTMS on motor cortical excitability and inhibition. Clin Neurophysiol. 2006 Dec;117(12):2584-96.

10 Sasaki N, Mizutani S, Kakuda W, Abo M. Comparison of the effects of high- and low-frequency repetitive transcranial magnetic stimulation on upper limb hemiparesis in the early phase of stroke. J Stroke Cerebrovasc Dis. 2013 May;22(4):413-8. 


\section{Case Reports in Neurology}

\begin{tabular}{|c|c|}
\hline DOI: 10.1159/000492381 & $\begin{array}{l}\text { (C) } 2018 \text { The Author(s). Published by S. Karger AG, Basel } \\
\text { www.karger.com/crn }\end{array}$ \\
\hline
\end{tabular}

Urushidani et al.: LF-rTMS/OT with Longitudinal fNIRS

11 Hosomi K, Morris S, Sakamoto T, Taguchi J, Maruo T, Kageyama Y et al. Daily repetitive transcranial magnetic stimulation for poststroke upper limb paresis in the subacute period. J Stroke Cerebrovasc Dis. 2016 Jul;25(7):1655-64.

12 Murase N, Duque J, Mazzocchio R, Cohen LG. Influence of interhemispheric interactions on motor function in chronic stroke. Ann Neurol. 2004 Mar;55(3):400-9.

13 Wei W, Bai L, Wang J, Dai R, Tong RK, Zhang Y et al. A longitudinal study of hand motor recovery after subacute stroke: a study combined FMRI with diffusion tensor imaging. PLoS One. 2013 May;8(5):e64154.
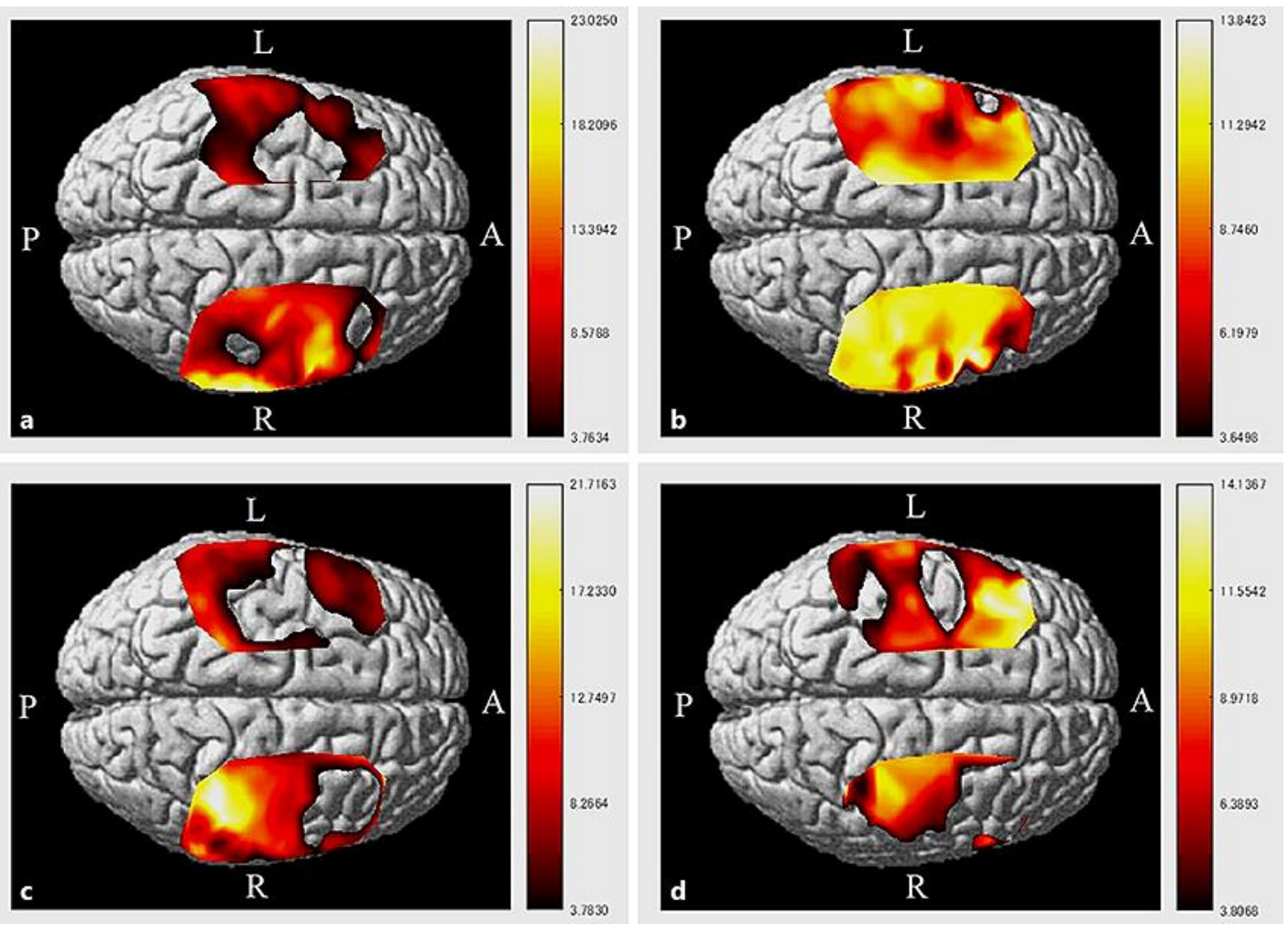

Fig. 1. HbO activation map during the tasks using NIRS-SPM (corrected, $p<0.01$ ). The patient had rightsided hemiparesis. a 28 days after stroke onset. Activation was identified in the nonaffected BA4 + BA6 areas. b 56 days after stroke onset. A high level of activation was detected in both hemispheres. c 109 days after stroke onset, pre-LF-rTMS/OT treatment. A high level of activation was found in the affected BA4 area. d 123 days after stroke onset, post-LF-rTMS/OT treatment. Note the decrease in activation on the nonaffected side and the increase in activation in the affected BA4 area. 
Table 1. Changes in test scores of upper limb motor function and cortical asymmetry as evaluated by fNIRS

\begin{tabular}{llll}
$\begin{array}{l}\text { At admission } \\
\text { (28th poststroke day) }\end{array}$ & $\begin{array}{l}\text { During admission } \\
\text { (56th poststroke day) }\end{array}$ & $\begin{array}{l}\text { Before rTMS } \\
(109 \text { th poststroke day) }\end{array}$ & $\begin{array}{l}\text { After rTMS } \\
\text { (123rd poststroke } \\
\text { day) }\end{array}$ \\
\hline 30 & 47 & 48 & 52 \\
6 & 34 & 35 & 37 \\
-0.419 & 0.100 & -0.190 & -0.073 \\
\hline
\end{tabular}

fNIRS, functional near-infrared spectroscopy; rTMS, repetitive transcranial magnetic stimulation; FMA, Fugl-Meyer Assessment; ARAT, Action Research Arm Test; LI, laterality index. 